\title{
Enhanced performance of thermographic asset monitoring by an interpolation-based correction algorithm
}

\author{
Aydin Boyaci ${ }^{1}$, Stephan Wildermuth ${ }^{1}$, Holger Kaul ${ }^{1}$, Tomas Kozel ${ }^{2}$ \\ ${ }^{1} A B B$ AG, Corporate Research, Wallstadter Str. 59, 68526 Ladenburg, Germany \\ ${ }^{2} A B B$ s.r.o., Vídeňská 117, 61900 Brno, Czech Republic
}

\begin{abstract}
Thermal condition monitoring of electrical assets requires an accurate temperature sensing to improve the lifetime and to prevent catastrophic overheating failures of the equipment. Low-resolution infrared (IR) cameras allow a permanent installation of temperature supervision in medium voltage switchgears. Besides their benefits of contactless measurement of temperature and capturing a large section of the electrical asset, low-resolution IR cameras lack in accuracy if the hot spot is spread over a small number of pixels in the thermal image. This contribution introduces an interpolation-based temperature correction algorithm to enhance the accuracy considerably of the temperature in the hot spot region. The feasibility of the proposed algorithm is demonstrated for a temperature rise test of a medium voltage switchgear under realistic conditions over the range of hot spot temperatures from $40^{\circ} \mathrm{C}$ to $100^{\circ} \mathrm{C}$.
\end{abstract}

Keywords: Low-resolution infrared camera, hot spot temperature measurement, medium voltage switchgear, condition monitoring

\section{Introduction}

Accurate online temperature measurement becomes increasingly important to perform condition monitoring and diagnostics of electrical assets to avoid catastrophic overheating events as well as to enhance the lifetime of the equipment. Infrared (IR) cameras allow for contactless measurement of temperature, offering a significant advantage compared to contacting methods like RFID- or SAW- sensors [1]. Low-resolution IR cameras (e.g. $32 \times 32$ pixels) with wide angle optics are ideally suited to capture a large part of the asset's internal structure with only one sensor, thus offering a commercially attractive solution with minimal impact on the dielectric properties [2]. In such a configuration, however, heated areas like bus bars or spouts only spread over a small number of pixels, in particular if located at the boundaries of the image. During measurements in real medium voltage (MV) switchgear panels our measurements indicate that the temperature of small-sized hot areas may get underestimated by the IR camera compared to a reference measurement with a conventional reference thermocouple. As this would limit the application range of the monitoring system, we have developed an interpolation-based temperature correction algorithm for low-resolution IR cameras which enhances the measurement accuracy of smallsized structures during online thermography.

\section{Temperature monitoring in MV switchgears \\ Reliable temperature condition monitoring of a MV switchgear comprises different} compartments and components (cf. Figure 1). Especially the contact locations are prone to high temperatures. Thus, the highest temperatures can be usually observed in the joints of the bus bar compartment which are the first choice to monitor. In addition to that, the temperature development of the circuit breaker spouts needs to be monitored. During a regular replacement of the breaker, a damage of the mechanical contact spring at the spouts might occur resulting in an increased electrical contact resistance and therefore high temperatures. At the bottom of the switchgear, the cable terminals are a further source of possibly high temperatures to be supervised since the cable connections can be incorrectly fastened in need of a cable rearrangement. 


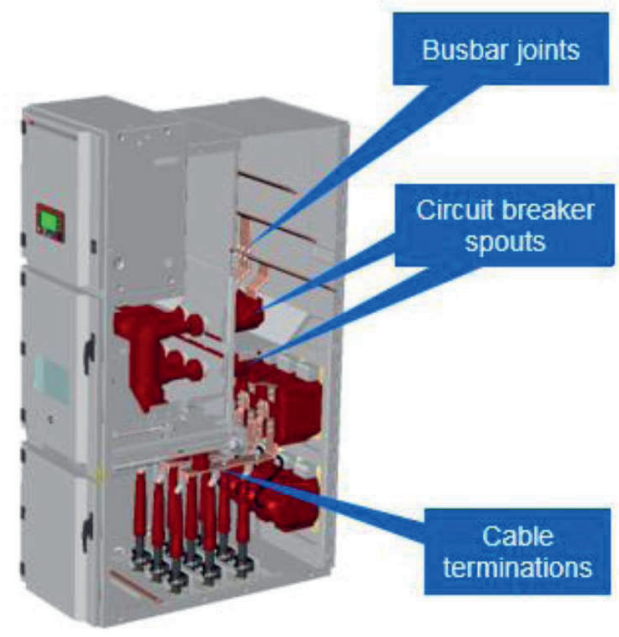

Figure 1: Schematic view of a MV switchgear panel. Temperature monitoring at the following locations: bus bar compartment (top), upper and lower contacts of the circuit breaker (middle), and cable compartment (bottom).

In a previous study [2], the feasibility of lowresolution IR cameras has been proved for the critical locations given in Fig. 1. The main advantage is that a single IR sensor is sufficient to monitor the temperature of the three phases for each location compared to an installation of three point-like sensors, i.e. one for each phase.

\section{Derivation of the interpolation-based temperature correction algorithm}

The temperature correction algorithm is derived beforehand from reference measurements with a large variety of hot area topologies. As outlined in Fig. 2a, a simple test rig is built up where the temperature $T_{\text {obj }}$ of an object can be controlled individually with a high degree of accuracy. The IR sensor is placed at different lateral and perpendicular positions to the object for capturing the temperature of hot spots of varying size as well as location in the image.

The derivation of the proposed algorithm consists of two main steps. Firstly, a spline interpolation is applied on the raw image of the infrared camera to increase the spatial image resolution which enhances the characterization of the hot spot in temperature and size (cf. Fig. $2 b$ ). This way, important features of the hot spot, in particular its size and initial temperature, are determined. Furthermore, the temperature distribution in close proximity to the hot area gets analyzed to extract input parameters for the correction routine and its derivation.
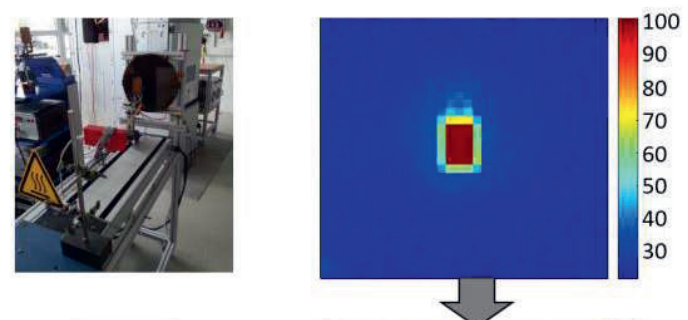

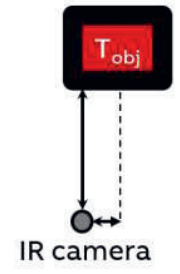

a)

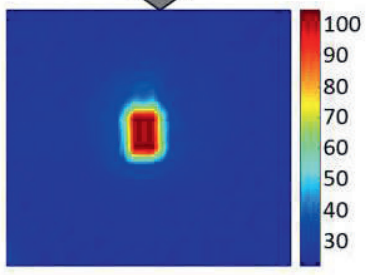

b)
Figure 2: Reference measurements for deriving the temperature correction algorithm of hot spots at different size and location in the image. a) Schematic measurement setup. b) Comparison between a raw image measured by a low-resolution IR camera and the spline interpolation of the raw image.

Secondly, the hot spot size-dependent algorithm is derived from the error between the measured IR temperature and the real object temperature $T_{\text {obj }}$ for different hot spot sizes. The curve fitting of the error leads to a global correction function over all hot spot sizes. Thus, this global correction function is dependent on the hot spot size and is valid for hot spot temperatures in the range from $40^{\circ} \mathrm{C}$ to $100^{\circ} \mathrm{C}$.

a)

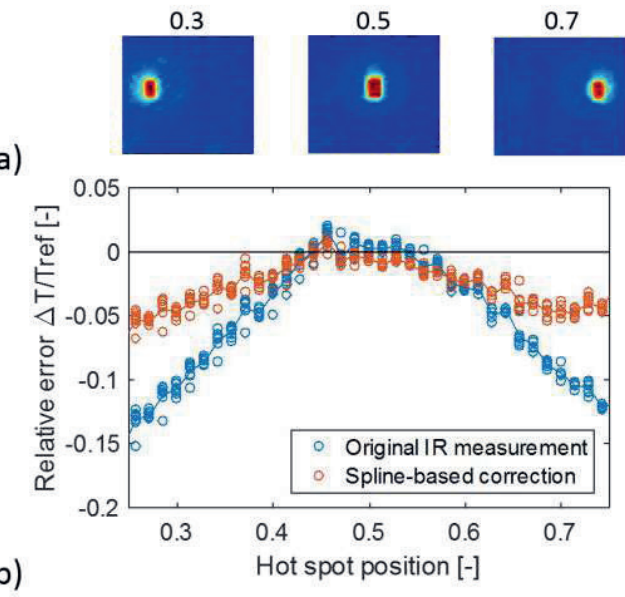

Figure 3: Temperature correction algorithm for a hot spot at different lateral positions in the image: a) Definition of hot spot position. b) Comparison of original IR-measured temperature and splinebased correction. 
In Fig. 3., the interpolation-based correction algorithm is applied for a hot spot temperature of $60^{\circ} \mathrm{C}$ and different lateral positions of the hot spot in the thermal image. The fisheye effect of the IR camera lens leads to a hot spot size reduction and thus to a larger underestimation of the temperature when the object reaches the boundaries of the image. As can be seen from Fig. $3 b$, the correction algorithm is capable to reduce considerably the temperature error measured by the IR camera, even in case of small-sized hot spots at the image boundaries.

\section{Temperature correction in a MV switchgear}

The interpolation-based correction algorithm is applied for a typical temperature rise test in a MV switchgear. The temperature rise test considers the heating up of the entire switchgear by applying the nominal current for several hours until a steady state temperature is reached. Representative for all mentioned monitoring locations, the section of the lower breaker spouts is discussed in detail below.

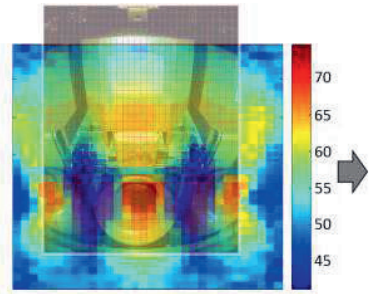

a)

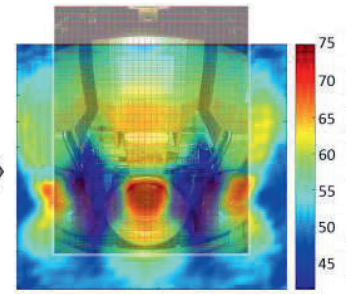

b)
Figure 4: Temperature distribution along the section of the lower breaker spouts of a MV switchgear (IR image and visible light image superimposed): a) Raw image measured by a lowresolution IR camera. b) Spline interpolation of raw image.

Fig. 4 demonstrates exemplary the spline interpolation of the thermal image for the section of the lower breaker spouts with the three phases at a steady state. Performing a spline interpolation at every time step of IR measurement, the hot spot temperature and size are determined as important features from the interpolated images. These features are used as input to the correction algorithm delivering the final temperature estimate of the hot spot. The corrected temperatures show a good agreement with the measurement of reference thermocouples (cf. Fig. 4), additionally installed at the considered hot spots of the switchgear.

We have demonstrated for a real temperature rise test, that the overall accuracy of lowresolution IR cameras can be improved to become better than $+-3^{\circ} \mathrm{C}$. By applying the interpolation-based correction algorithm, the feasibility has been proved to use lowresolution IR cameras in future applications for condition monitoring of switchgears.
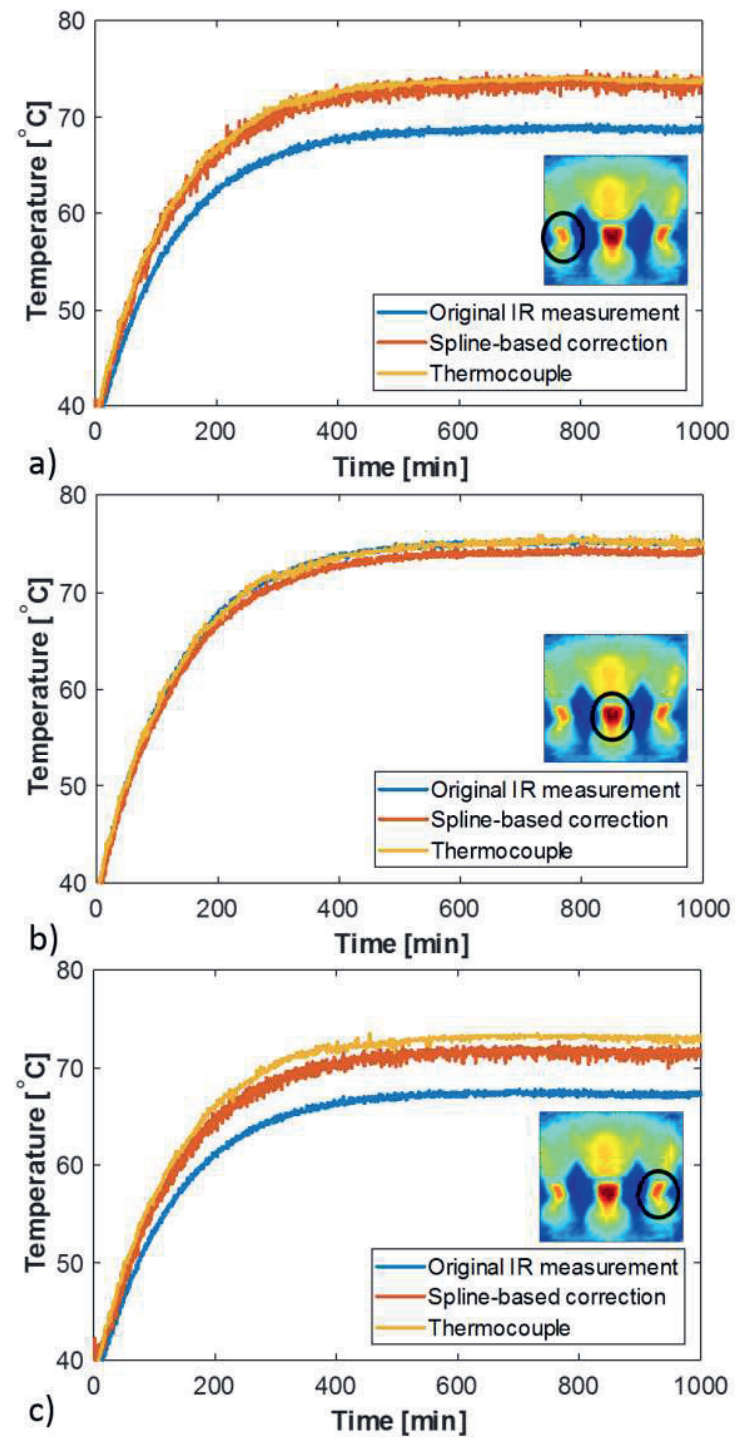

Figure 4: Comparison of original IR-measured temperature, spline-based correction and reference thermocouple temperature along the section of the lower breaker spouts of a MV switchgear at different phases: a) Left phase. b) Middle phase. c) Right phase.

\section{Conclusion}

The combination of the proposed temperature correction algorithm and a spline interpolation offers more accurate thermographic measurement by low-resolution IR cameras and makes these systems applicable for an even wider range of online condition monitoring applications in electrical assets. 


\section{Literature}

[1] J.C. Andle, S. Sabah, D. S. Stevens, S. J. Jumani, M. Baier, B. W.A. Wall, T. Martens, R. Gruenwald, "Temperature Monitoring System Using Passive Wireless Sensors for Switchgear and Power Grid Asset Management", MNCCIGRE/CIRED Malaysia, T\&D Asset

Management Workshop (2010)

[2] S. Wildermuth, P. Szasz, J. Gebhardt, H. Kaul, K. König, "Infrared temperature sensing in electrical equipment by low-cost IR cameras", VDE-Hochspannungstechnik, Berlin (2010) 